\title{
Die zweimalige Kaiserschnitt-Entbindung durch den Zürcher Stadtwundarzt Johann Jakob Locher von 1817/18
}

Christoph Mörgeli und Stefan Schulz

Prof. Dr. med. Urs Boschung zum 50. Geburtstag

\section{Summary}

Caesarean delivery was rarely practised in the early nineteenth century and was considered highly dangerous, being both technically and morally controversial. In view of this, Johann Jakob Locher's performance of two consecutive caesareans attracted international attention. Not only contemporary printed literature, but also the archive material and specimens presented in this journal for the first time provide a uniquely detailed account of the operations. The University of Zurich's Institute and Museum of the History of Medicine were able to acquire the preserved pelvis of the patient in 1983.

\section{Zusammenfassung}

Der Kaiserschnitt wurde Anfang des 19. Jahrhunderts zu den selten ausgeführten, äusserst riskanten Operationen gerechnet. Er war sowohl pragmatisch-technisch als auch moralisch heftig umstritten. In dieser Situation erregte eine zweimalige Kaiserschnittentbindung durch den Zürcher Stadtwundarzt Johann Jakob Locher internationales Aufsehen. Die Geschichte dieser geburtshilflichen Sensation der Jahre 1817 und 1818 ist nicht nur durch die gedruckte Literatur, sondern auch durch hier erstmals vorgestellte Ar-

PD Dr. phil. Christoph Mörgeli, Medizinhistorisches Institut und Museum der Universität Zürich, Rämistrasse 71, CH-8006 Zürich - Dr. med. Stefan Schulz, Institut für Geschichte der Medizin und Medizinhistorische Sammlung der Ruhr-Universität Bochum, Markstrasse 258a, D-44799 Bochum 


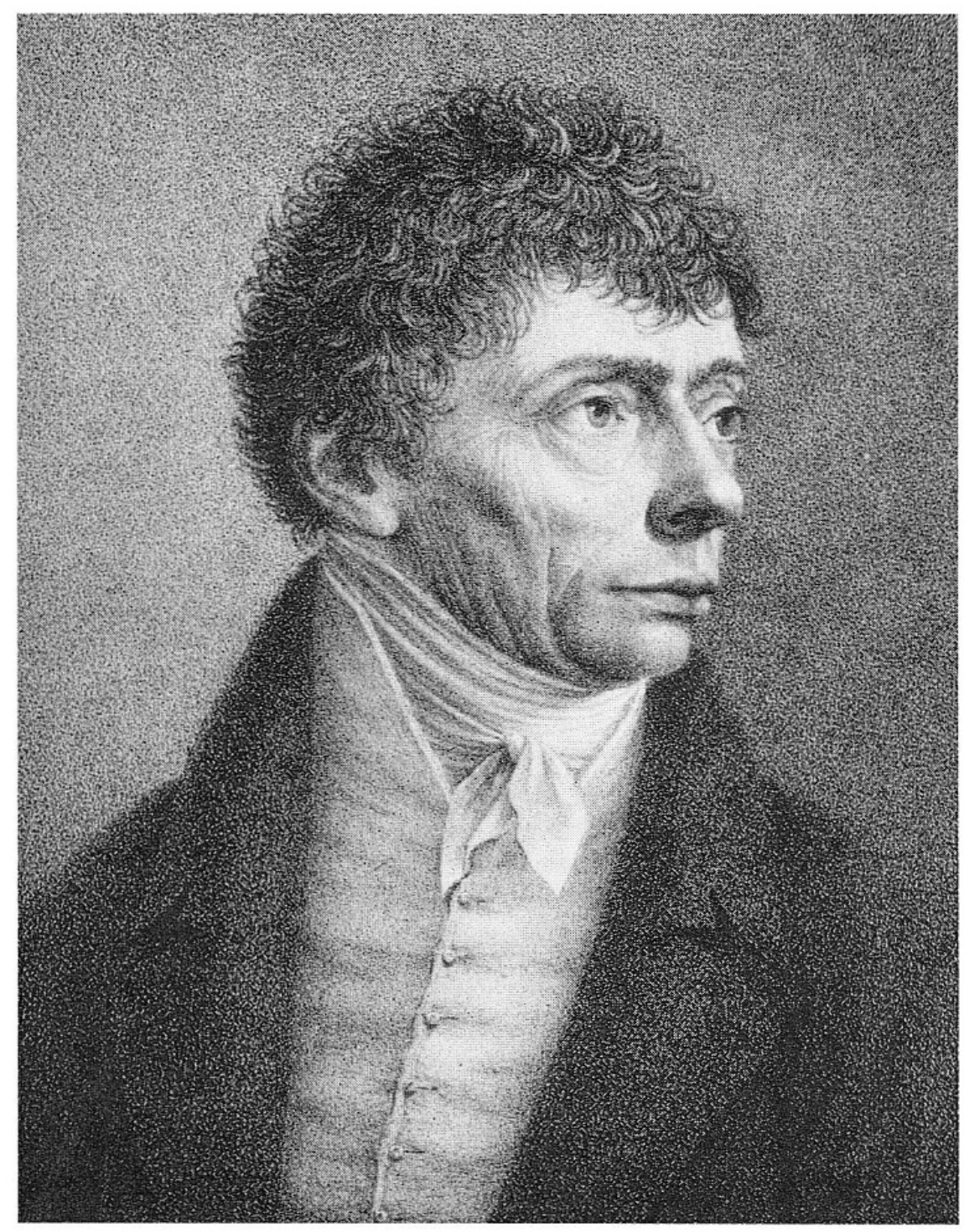

Abb. 1. Johann Jakob Locher (1771-1832), Stadtschnittarzt von Zürich und Ehrenmitglied der Medical and Chirurgical Society von London. Medizinhistorisches Institut und Museum der Universität Zürich. 
chivalien und Realien in einmalig vollständiger Weise dokumentiert. Das Beckenpräparat der Patientin gelangte 1983 in den Besitz des Medizinhistorischen Instituts und Museums der Universität Zürich.

Im Jahre 1820 ernannte die angesehene «Medical and Chirurgical Society» zu London den Zürcher Stadtschnittarzt Dr. med. Johann Jakob Locher zu ihrem Ehrenmitglied. Sie würdigte damit Lochers zweimalige KaiserschnittEntbindung an derselben Patientin. Wie aber war die Nachricht der aufsehenerregenden Eingriffe von Zürich ins ferne London gelangt? Am 11. September 1818 erwähnten die «Monatlichen Nachrichten Schweizerischer Neuheiten» den «von Hrn. Dr. Locher innerhalb von 15 Monathen an der nähmlichen Person zweymahl verübten Kaiserschnitt, der beide Mahl für die Mutter mit gutem Erfolg, und das erste Mahl mit nicht minder erwünschtem für das lebende Kind von Statten ging» ${ }^{1}$. An der Herbstversammlung der Medizinisch-chirurgischen Gesellschaft des Kantons Zürich von 1818 verlas Locher ein 41seitiges Manuskript über den interessanten Fall². Schon nach der ersten, für Mutter und Kind erfolgreich verlaufenen Operation hatte Johann Abraham Albers in Bremen, ein Freund Lochers aus gemeinsamer Jenaer Studienzeit, den Zürcher Kollegen um Zusendung eines Berichtes gebeten; die Richtigkeit von Lochers schriftlichen Ausführungen bestätigte der Zürcher Stadtarzt (Archiater) David Rahn, die Echtheit der Unterschrift Staatsschreiber Heinrich Hottinger. Albers gehörte zu den fleissigsten medizinischen Autoren seiner Zeit und bemühte sich mit zahlreichen Übersetzungen um den Erfahrungsaustausch zwischen englisch- und deutschsprachigen Ärzten $^{3}$. Im neunten Band der «Medico-Chirurgical Transactions» der Medizinisch-chirurgischen Gesellschaft zu London erschien der am 5. Januar 1818 eingegangene Bericht von Lochers erster Kaiserschnittoperation in Albers' Übersetzung ${ }^{4}$. In einer kurzen Vorrede erwähnte Albers den «erstaunlichen Umstand», dass in Grossbritannien, wo so viele Operationen mit glücklichem Erfolg ausgeführt würden, gerade der Kaiserschnitt auffallend wenig ver-

1 Monatliche Nachrichten Schweizerischer Neuheiten, September 1818, S. 116.

2 Locher, J. J.: Geschichte eines zweymal an der nämlichen Person verrichteten Kaiserschnitts, Mskr., o. J. [1818], Staatsarchiv Zürich W 52.11.2.

3 Schmidt, G. H.; Wilckens, H.: Biographische Skizzen verstorbener Bremischer Aerzte und Naturforscher, Bremen 1844, S. 199-237. Bynum, W. F.: Johann Abraham Albers (1772-1821) and American medicine, in: Journal of the History of Medicine 23 (1968), pp. 50-62.

4 History of a case of caesarean operation, in which the lives of the mother and child were both preserved, by J. J. Locher, M. D., town physician of Zurich, communicated by J. A. Albers, M. D., of Bremen, in: Medico-Chirurgical Transactions, published by the Medical and Chirurgical Society of London, vol. 9 (1818), pp. 11-25. 


\section{Anribisitin}

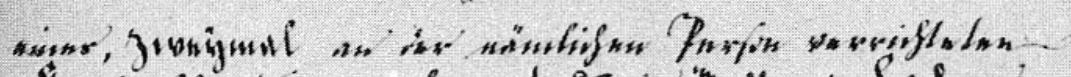

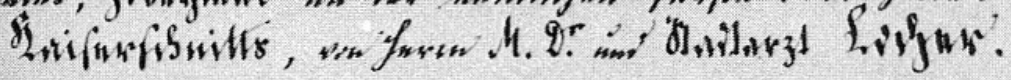

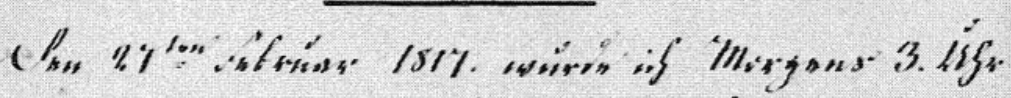

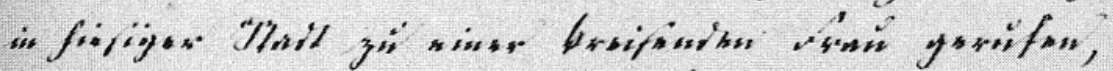

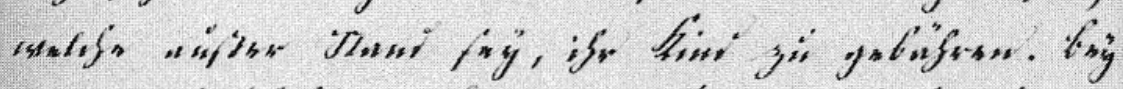

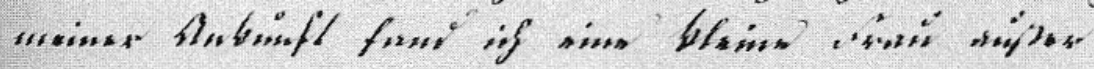

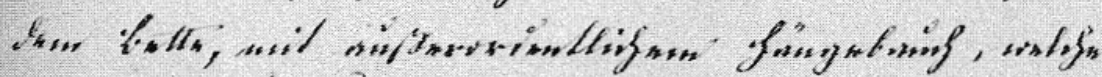

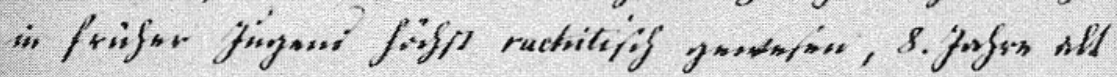

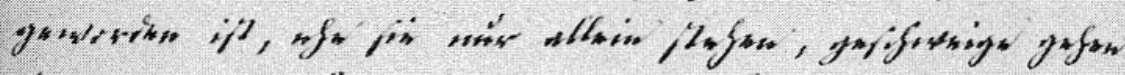

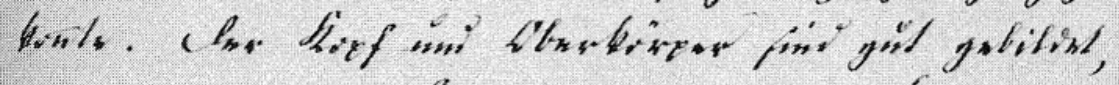

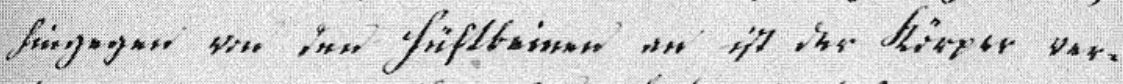

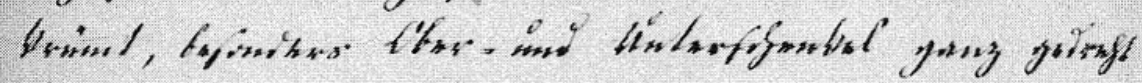

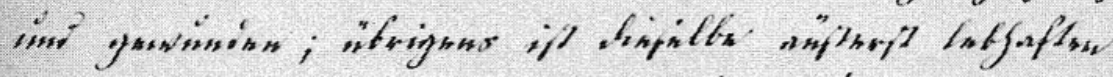

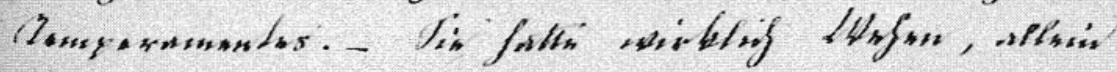

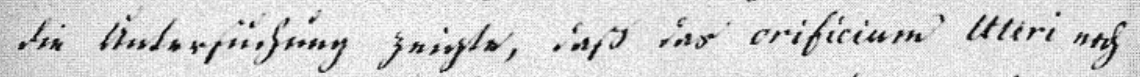

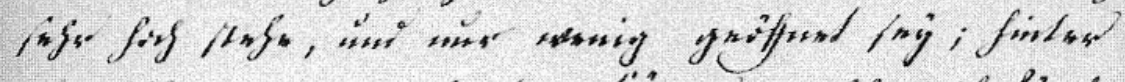

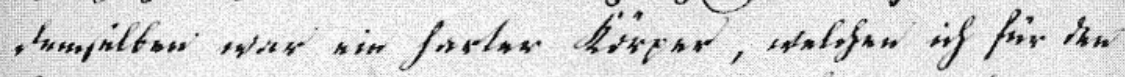

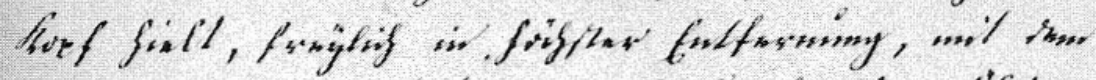

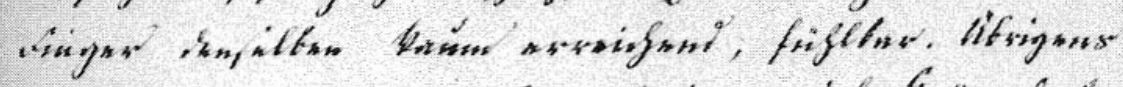

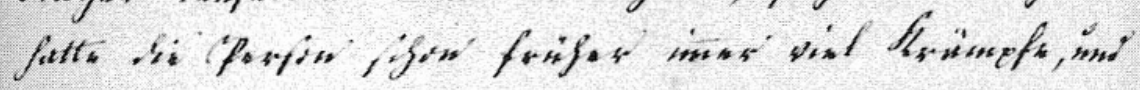

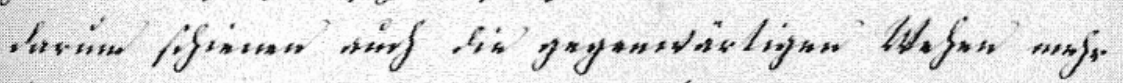

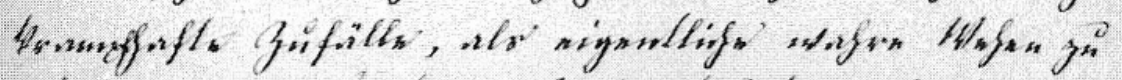

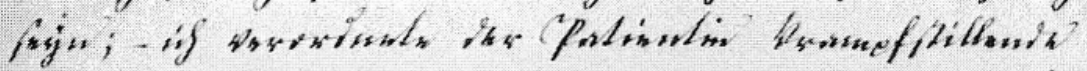

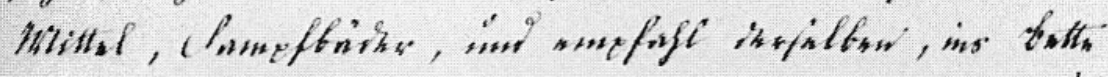

Abb. 2. Lochers Manuskript der «Geschichte eines zweymal an der nämlichen Person verrichteten Kaiserschnitts», o. J. [1818], Staatsarchiv Zürich W 52.11.2. 


\title{
HISTORY OF A CASE
}

OF

\section{CESAREAN OPERATION,}

\author{
IN WHICH THE
}

LIVES OF THE MOTHER AND CHILD WERE BOTH PRESERVED.

By J. J. LOCHER, M.D.

TOWN PHYSICIAN OF ZURICH.

COMMUNICATED

By J. A. ALBERS, M.D.

OF BREMEN.

WITH A FEW PRELIMINARY OBSERVATIONS BY THE LATTER.

Read January 5, 1818.

Abb. 3. Titelblatt der Mitteilung über einen gelungenen Kaiserschnitt durch Johann Jakob Locher und Johann Abraham Albers in den «Medico-Chirurgical Transactions» in London, Bd. 11, 1818. 
sucht werde. 1798 habe Albers anlässlich seines Aufenthaltes in London vom bedeutenden Wundarzt John Haighton erfahren, dass in England das Leben der Mutter nach erfolgtem Kaiserschnitt noch in keinem einzigen Fall habe gerettet werden können; dasselbe versicherte ihm 1817 Professor William Lawrence im Antwortschreiben auf eine entsprechende Nachfrage ${ }^{5}$. Lochers geburtshilfliche Leistung erschien seinenZeitgenossen jedenfalls ganz ausserordentlich und steigerte sich zur Sensation, als er den Kaiserschnitt an derselben Frau wiederholte und dabei zwar eine Totgeburt entband, das Leben der Mutter aber zumindest für sieben Wochen retten konnte. In einer Fortsetzung des ersten Aufsatzes machte Albers 1821 auch diesen zweiten Eingriff im Organ der Medizinisch-Chirurgischen Gesellschaft Londons bekannt ${ }^{6}$.

\section{Häufigkeit und Prognose des Kaiserschnitts}

Nach Auskunft namhafter deutschsprachiger Geburtshelfer wendeten die Wundärzte auf dem Kontinent den Kaiserschnitt häufiger an als ihre Kollegen in Grossbritannien ${ }^{7}$. Absolut betrachtet griffen aber auch sie nur sehr selten zu dieser riskanten Operation: Karl Kayser (1811-1870), der spätere Professor für Statistik und Staatsökonomie in Kopenhagen ${ }^{8}$, berichtet in seiner Doktorarbeit aus dem Jahre 1841, dass in ganz Mitteleuropa zwischen 1750 und 1800 weniger als 120 Operationen nachweisbar sind ${ }^{9}$. Doch diese exakt anmutende Angabe soll nicht darüber hinwegtäuschen, dass wir die genauen quantitativen Verhältnisse für die Zeit vor 1800 kaum mehr rekonstruieren

5 Zur besonders schlechten Prognose des Kaiserschnitts in England vgl. z. B. die von [Osiander, F. B.]: Handbuch der Entbindungskunst. Bd. 3, bearb. v. J. F. Osiander, Tübingen 1825, S. 199 zitierte Literatur, beispielsweise J. Burns, Principles of midwifery, London 1814, S. 365. Nach Burns wurden bis 1814 in Grossbritannien nur 21 Kaiserschnittoperationen durchgeführt, wobei nur eine Mutter überlebte; vgl. auch Osborn, W.: Versuch über die Geburtshülfe in natürlichen und schweren Geburten, aus dem Englischen von C.F. Michaelis, Liegnitz 1794, S. 312ff., der von 11 englischen Operationen berichtet, die sämtlich mit dem Tod der Mutter endeten (die englische Originalpublikation erschien 1792); vgl. auch Michaelis, A.: Geschichtliche Bemerkungen über den Kaiserschnitt..., in: Abhandlungen aus dem Gebiete der Geburtshilfe, hrsg. von A. Michaelis, Kiel 1833, S. 34-216, bes. S. 132-139; Naegele, H. F.: Lehrbuch der Geburtshülfe. 2. Teil, Mainz 1845, S. 109/110.

6 History of a second caesarean operation, performed on the patient, whose case is related in the ninth volume of the Society's Transactions, by J. J. Locher, M. D. of Zurich, communicated by J. A. Albers, M. D. of Bremen, in: Medico-Chirurgical Transactions, vol. 11 (1821), pp. 182-199.

7 Vgl. z. B. Osiander (1825, wie Fussnote 5), S. 199 und Michaelis (1833, wie Fussnote 5), S. 131-135.

8 Vgl. Biographisches Lexikon der hervorragenden Ärzte aller Zeiten und Länder, hrsg. v. A. Hirsch, Bd. 3, Berlin, Wien 1931, S. 494.

9 Kayser, K.: De eventu sectionis caesareae, Kopenhagen 1841, S. 99 (von 1750 bis 1800: 37 Fälle, in denen die Mutter den Kaiserschnitt überlebte, 80 Fälle mit tödlichem Ausgang für die Mutter). 
können, obgleich uns zahlreiche Fallsammlungen zum Kaiserschnitt zur Verfügung stehen ${ }^{10}$, deren Traditionskette bis ins 16. Jahrhundert zurückreicht. Die Qualität der Literaturdaten ist bis in das frühe 19. Jahrhundert hinein einfach zu schlecht.

Die erste Monographie über den Kaiserschnitt veröffentlichte 1581 der französische Leibarzt des Herzogs von Savoyen und spätere Wundarzt in Paris, François Rousset (1535-1590? $)^{11}$. In dieser Schrift lobt und feiert Rousset den Kaiserschnitt in höchsten Tönen als das beste Mittel, um bei schweren Geburten Mutter und Kind zu retten. Die von ihm zusammengestellten wenigen Fälle erregten höchstes Aufsehen, obwohl er keinen einzigen Kaiserschnitt selbst operiert, wahrscheinlich nicht einmal eine Operation mit eigenen Augen gesehen hatte. Das Buch wurde schon kurz nach dem Erscheinen ins Deutsche und dann ins Lateinische übersetz $\mathrm{t}^{12}$. In der Nachfolge Roussets ergänzten und aktualisierten in der Folgezeit verschiedene Ärzte die bereits bekannten Zusammenstellungen. Doch auch die neuen Fallsammlungen waren oftmals mangelhaft ${ }^{13}$ : häufig gaben die Autoren weder den genauen Ort und die genaue Zeit des Eingriffes, noch den Namen ihrer Patientinnen an, manchmal wurde sogar nur lückenhaft über die Art des Eingriffes

10 Gute Überblicke über die in der geburtshilflichen Fachliteratur des frühen 19. Jahrhunderts diskutierten Fallsammlungen finden sich z. B. bei Michaelis (1833, wie Fussnote 5) sowie bei Kayser (1841, wie Fussnote 9).

11 Rousset, F.: De Partu Caesareo... Jetz und erstmals von Meyster Frantzen Rousset Medico frantzoesisch beschrieben... nun erstmals vom Doctore Melchiore Sebizio Silesio auss frantzoesischer Sprach in Tuetsche gebracht, Strassburg 1583; die Erstausgabe «Traitte nouveau de l'hysterotomotocia... » erschien 1581 in Paris. Zur Geschichte des Kaiserschnittes im allg. vgl. z. B. Fasbender, H.: Geschichte der Geburtshilfe, Jena 1906 (Reprint Hildesheim 1964), bes. S. 979-1010; Weindler, F.: Der Kaiserschnitt. Nach den ältesten Überlieferungen unter Zugrundelegen von 18 Geburtsdarstellungen, in: Janus (Leiden) 20 (1915), S. 1-40 u. Tafel I-IX; Hofschläger, R.: Der Ursprung des Kaiserschnitts (I) und (II), in: Sudhoffs Archiv für Geschichte der Medizin und der Naturwissenschaften 36 (1943), S. 284-299 sowie 37 (1953), S. 77-92; Quecke, K.: Über die Anfänge des Kaiserschnittes, ders.: Der Kaiserschnitt an der Toten, ders.: Der Kaiserschnitt an der Lebenden, in: Ciba-Zeitschrift (Basel) 11 (Nr. 128) (1952), S. 4706-4710, S. 4711-4716 u. S. 4717-4725; Müller, C.: Die Schnittentbindung seit der Einführung der Porroschen Operation (1876), in: Ciba-Zeitschrift (Basel) 11 (Nr. 128) (1952), S. 4727-4735; Bazala, V.: Zur Geschichte des Kaiserschnitts. Idee und Wirklichkeit, in: Die Grünenthal Waage 4 (Nr. 2) (1965), S. 61-72; Zglinicki, F. v.: Geburt: Eine Kulturgeschichte in Bildern. Braunschweig 1983, S. 125-140; Gabert, H. A.; Bey, M.: History and Development of Cesarean Operation, in: Obstetrics and Gynecology Clinics of North America 15 (1988), S. 591-605; Blumenfeld-Kosinski, R.: Not of Women Born, London 1990; Boley, J. P.: The History of Caesarean Section, in: The Journal of the Canadian Medical Association 145 (1991), S. 319-322; Kloeppel, A. B.: Kraniotomie oder Kaiserschnitt im 19. Jahrhundert, Med. Diss. Hannover 1992; Schulz, S.: In schweren Kintsnöthen. Zur Geschichte des ärztlichen Handelns, in: TW Gynäkologie 6 (1993), S. 137-146; Schäfer, D.: Sectio in mortua. Med. Diss. Freiburg 1995.

12 Vgl. Quecke, K.: François Rousset und die erste Monographie über den Kaiserschnitt, in: Centaurus 2 (1951-1953), S. 349-363.

13 Vgl. z. B. Michaelis (1833, wie Fussnote 5); Kayser (1841, wie Fussnote 9). 
selbst informiert. Zudem wurden die Kaiserschnittfälle zunächst nicht mit der Absicht gesammelt, eine Statistik im heutigen Sinne zu liefern - das uns vertraute statistische Denken lässt sich erst nach 1800 nachweisen ${ }^{14}$. Die Ärzte und Wundärzte verfolgten zunächst andere Ziele: Rousset und anderen frühen Autoren ging es beispielsweise um den Nachweis, dass die umstrittene Operation nicht zwangsläufig mit dem Tode der Mutter und des Kindes enden musste, so dass in den frühen Fallsammlungen keine oder nur sehr wenige tödlich endende Operationen auftauchen ${ }^{15}$. Bei oberflächlicher Analyse ergibt sich daher sogar der falsche Eindruck, dass die Erfolgsquote der Kaiserschnittoperationen vom 16. zum 19. Jahrhundert nicht besser, sondern schlechter geworden $\operatorname{se}^{16}$. Ausserdem vermuteten schon Zeitgenossen Lochers - wahrscheinlich zu Recht -, dass die bekannten Zahlen noch aus einem weiteren Grund nicht die tatsächlichen Verhältnisse widerspiegeln: es werde nämlich jede Operation veröffentlicht, die die Kreissende überlebte, nicht aber jeder Fall mit tödlichem Ausgang ${ }^{17}$.

In der ersten Hälfte des 19. Jahrhunderts versuchten verschiedene Autoren, die alten Fallsammlungen unter strengen Qualitätsvorgaben neu zu bewerten: unglaubwürdige Fälle sollten endlich ausgesondert, doppelte Schilderungen erkannt und letztendlich eine realistische quantitative Bewertung des mütterlichen und kindlichen Risikos ermöglicht werden ${ }^{18}$. Die zuverlässigste Arbeit aus dieser Zeit ist die schon erwähnte Dissertation «De eventu sectionis caesareae» von Kayser. Dieser extrahierte für die Zeit bis 1839 insgesamt 338 Fälle aus der Literatur, die er als «sichere» Kaiserschnittoperationen bewertete. 210 Frauen (62\%) verstarben während oder kurz nach den Eingriffen. In den Fallschilderungen wurden 281 Kinder erwähnt, von denen $86(31 \%)$ nicht überlebten ${ }^{19}$.

Die erste Operation des Zürcher Stadtschnittarztes Johann Jakob Locher aus dem Jahre 1817 trägt die Nummer 50 in Kaysers Gruppe der «glücklich» verlaufenen Fälle ${ }^{20}$. Den zweiten Eingriff aus dem Jahre 1818 bezeichnet Kayser allerdings nicht als «Sectio caesarea», sondern als «Gastrotomia», da

14 Vgl. z. B. Müller, I.: Bericht am Krankenbett - Zum Beginn der medizinischen Dokumentation und Statistik, in: Dokumentation und Archivierung im Krankenhaus, hrsg. v. G. Hierholzer und P.-M. Hax. Stuttgart, New-York 1992, S. 1-7 (=Traumatologie aktuell 7).

15 Michaelis (1833, wie Fussnote 5), S. 36; vgl. auch Naegele (1845, wie Fussnote 5), S. 108-110.

16 Vgl. Michaelis (1833, wie Fussnote 5), S. 40-41.

17 Vgl. Naegele, F. K.: De jure vitae et necis quod competit medico in partu. Heidelberg 1826, S. 7/8; Osiander (1825, wie Fussnote 5), S. 198; vgl. aber Osiander, F. B.: Handbuch der Entbindungskunst. Bd. 2, 2. Abteilung, Tübingen 1821, S. 329, der dieses Urteil nicht bestätigt.

18 Vgl. Naegele (1845, wie Fussnote 5), S. 108-110; vgl. auch Fasbender (1906, wie Fussnote 11), S. 996-997.

19 Kayser (1841, wie Fussnote 9), S. 99-100.

20 Kayser (1841, wie Fussnote 9), S. 25. 
während der Operation zwar die Bauchdecken, nicht aber die in den Wehen bereits aufgerissene Gebärmutter eröffnet worden waren ${ }^{21}$. Die von Albers und Locher benutzte Terminologie - sie hatten ja übereinstimmend in beiden Fällen von einem «Kaiserschnitt» geschrieben - wurde also nicht allgemein akzeptiert.

Der Kaiserschnitt war zu Lochers Zeit schon an sich eine ziemlich seltene Operation - mehrmalige Eingriffe galten als absolute Rarität. Michaelis hält aus der Zeit vor 1817 weniger als 10 Fälle für glaubwürdig. Einer dieser Eingriffe wurde bereits in Zürich vorgenommen ${ }^{22}$.

\section{Erster Kaiserschnitt: Leben von Mutter und Kind gerettet}

Am 27. Februar des Jahres 1817 wurde Stadtschnittarzt Johann Jakob Locher morgens drei Uhr zu einer kreissenden Frau mit ausserordentlichem Hängebauch gerufen, deren Namen und nähere Umstände er uns nicht überliefert hat. Die Akten der Zürcher Archive belegen aber einwandfrei, dass es sich dabei um die 27jährige Elisabeth Bröckli, geborene Näf von Zürich, gehandelt hat. Ihr 1785 geborener Gatte Jakob, von Beruf Schneider, stammte aus Glattfelden und hatte sich als «Ansässe» in Zürich eingekauft ${ }^{23}$. Wie Locher sofort feststellte, waren Oberkörper und Kopf der kleinwüchsigen Frau gut gebildet, jedoch ihre unteren Extremitäten vom Hüftbein an entstellt; speziell die Ober- und Unterschenkel erschienen ganz verdreht und gewunden. Von der geistig lebhaften, temperamentvollen Schwangeren erfuhr der Arzt, dass sie vor dem achten Lebensjahr nicht allein habe stehen, geschweige denn gehen können. Die einige Stunden später vorgenommene Untersuchung zeigte bei anhaltend starken Wehen ein durch Rachitis völlig defor-

21 Vgl. dazu z. B.Weidemann, J. P.: Entwurf der Geburtshilfe, Mainz 1808, S. 254; Osiander (1821, wie Fussnote 17), S. 302-303; Osiander (1825, wie Fussnote 5), S. 76; Busch, D. W. H.: Die theoretische und practische Geburtskunde durch Abbildungen erläutert, Berlin 1838, S. 419; Kraus, L. A.: Kritisch-etymologisches medicinisches Lexikon... Göttingen 1844: Sectio caesarea $=$ Kaiserschnitt $=$ Hysterotomotocia (Stichwort: caesareus, S. 182; sectio, S. 939); vgl. dagegen Heister, L.: Chirurgie, in welcher alles, was zur Wundarzney gehöret..., Nürnberg 1763 (Reprint Osnabrück 1981), S. 701 u. 703/4, der den Terminus «kayserlicher Schnitt» wie Locher und Albers verwendet.

22 Michaelis (1833, wie Fussnote 5), S. 47-74. In Zürich wurde 1801 eine Frau durch einen Kaiserschnitt entbunden, die bereits 1797 in Basel operiert worden war, wo sie auch 1805 an der dritten Operation verstarb (S. 47). Lochers Operationen listet Michaelis unter der Nummer 3 in der Gruppe der zweimaligen Operationen mit tödlichem Ausgang für die Mutter auf (S. 69).

23 Staatsarchiv Zürich: Acta der Wundgschau 1817-1832, Bd. 8, H I 334, S. 28 f. Stadtarchiv Zürich: Totenbuch Spital 1797-1823, VIII C 56 (9. Juli 1818), sowie Totenbuch Predigern 1810-1862, VIII C 43 (9. Juli 1818). 
miertes Becken, in das der Kopf des Kindes unmöglich eintreten konnte, «da nach aller Untersuchung das Becken in seiner Conjugata nicht mehr als 2, höchstens $2 \frac{1}{2}$ Zoll hatte, und die übrigen Diameter in ähnlichem Verhältnisse standen». Die Beckenmasse hatte Locher nach den vorliegenden Quellen durch eine manuelle Untersuchung, also ohne instrumentelle Hilfsmittel, bestimmt ${ }^{24}$.

Locher ersuchte den Geburtshelfer Conrad Spöndli um Unterstützung; als weder die Rückenlage im Bett noch der Wechsel auf den Gebärstuhl den Geburtsakt voranbrachten, führte Locher einen Schenkel der von ihm modifizierten Geburtszange ein, der sich nach grossen Anstrengungen in völlig schiefer Stellung befand. Das für die Patientin äusserst schmerzhafte Unterfangen, auch den zweiten Schenkel einzuführen, musste bald aufgegeben werden. Die Perforation wurde verworfen, da Locher es als unmöglich beurteilte, die entsprechenden Instrumente ohne Verletzung innerer Teile einzubringen. Da sowohl die Mutter wie die Ärzte die Bewegungen des Kindes wahrnahmen, indizierten sie den Kaiserschnitt «in der Überzeugung, dass dadurch wenigstens das Leben eines gerettet werden könne»: «Die nächsten Anverwandten, welche gegenwärtig waren, wurden also mit diesem Entschluss bekannt gemacht, und, auf Genehmigung derselben, er auch der Gebährenden angezeigt. Der Entschluss ward von dieser, nach wenigem Einreden, bald gefasst, und von uns der Apparat und alles Nöthige vorbereitet.. ${ }^{25}$ Abends fünf Uhr nahm Locher den Eingriff im Wohnhaus der Familie Bröckli in Gegenwart mehrerer Kollegen und Gehilfen vor, wobei die Frau in üblicher Rückenlage «gehörig festgehalten» wurde. Der Bauchschnitt entlang der Linea alba betrug 24 bis $30 \mathrm{~cm}$; ein vordrängendes Stück Dünndarm konnte mittels einem mit Fett bestrichenen Läppchen zurückgehalten werden. Nach einem 18 bis $24 \mathrm{~cm}$ langem Schnitt durch den Uterus konnte ein Mädchen entwickelt und entbunden werden. Zur grossen Freude der Mutter und der Umstehenden schrie es heftig und musste nicht im geringsten belebt werden. Nach Herausnahme der Plazenta trat aus dem Uterus eine wel-

24 Damit mass die «Conjugata» nach Lochers Befund zwischen 5 und 6,5 $\mathrm{cm}$ (Umrechnung nach der Tabelle «Europäische Längeneinheiten im 1. Drittel des 19. Jahrhunderts`, in:Trapp, W., Kleines Handbuch der Masse, Zahlen, Gewichte und der Zeitrechnung, Stuttgart 1992, S. 225-226 und Duber, A.-M., Masse und Gewichte im Staat Luzern und in der alten Eidgenossenschaft, Luzern 1975, S. 9-21). Das Rechenergebnis wurde auf 0,5 cm gerundet; der Zürcher Fuss [ $=12$ Zürcher Zoll] hatte eine Länge von $30,16 \mathrm{~cm}$, der Nürnberger Fuss von $30,38 \mathrm{~cm}$, der Pariser Fuss von 32,48 cm, der Rheinische Fuss von $31,39 \mathrm{~cm}$; vgl. unten: «Beckenbeschreibung». Die instrumentelle Beckenmessung war erst gegen Ende des 18. Jahrhunderts entwickelt worden; vgl. z. B. Wengen-Dörig, D.: Die Geschichte der manuellen und instrumentellen Beckenmessung 1650-1886, Med. Diss. Zürich 1987.

25 Locher, J. J.: Geschichte eines zweymal an der nämlichen Person verrichteten Kaiserschnitts, Mskr., Staatsarchiv Zürich W 52.11.2,27. Februar 1817, o. J. [1818], S. 5. 


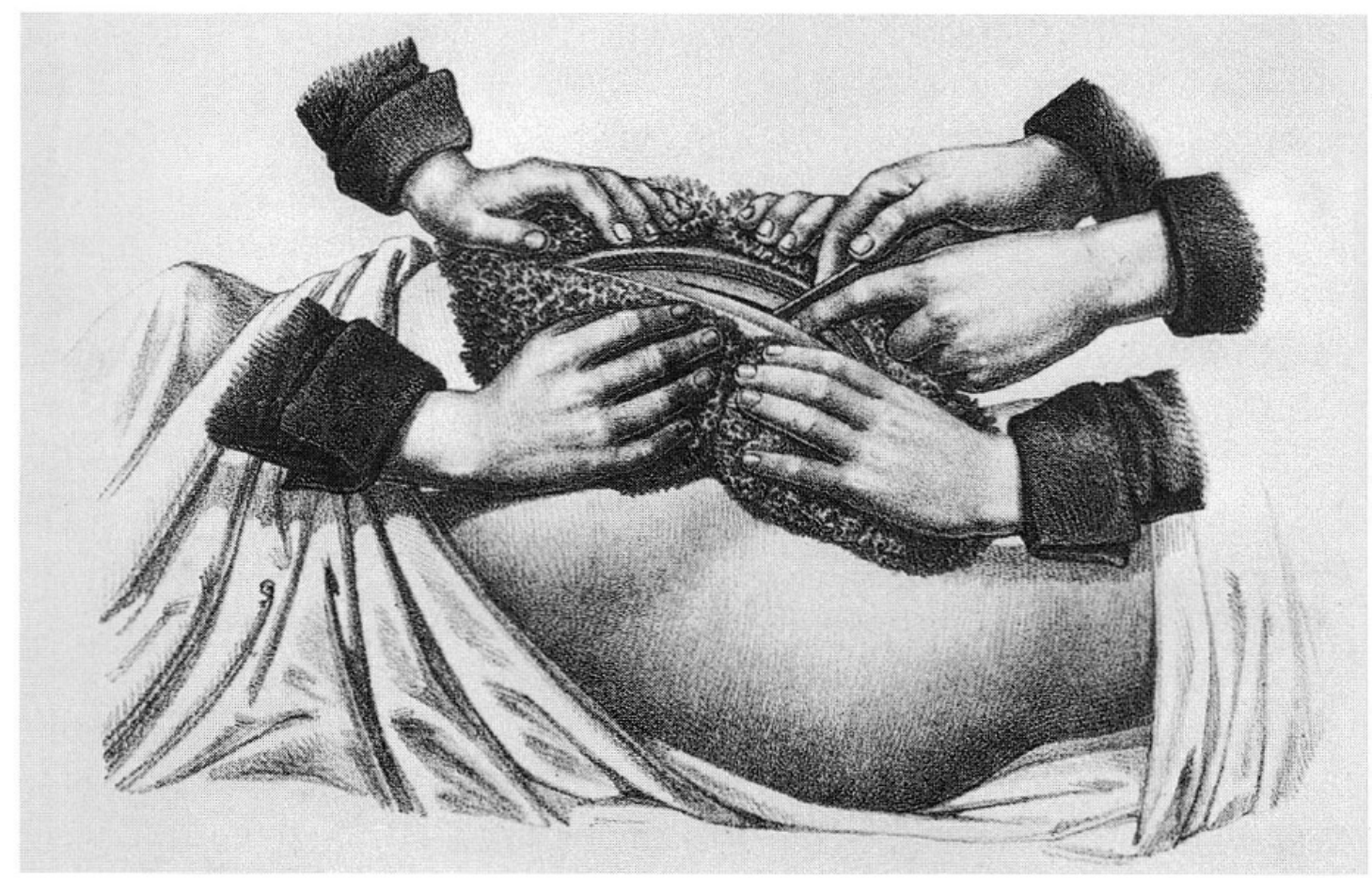

Abb. 4. Kaiserschnitt durch Bauchfell und Gebärmutter, wobei ein Auseinanderklaffen der Wunde und dadurch provoziertes Vortreten von Darmschlingen mit Schwämmen vermieden werden soll. Eduard Caspar Jacob von Siebolds Abbildungen aus dem gesammten Gebiet der theoretisch-praktischen Geburtshülfe, Berlin 1829, Tafel LVIII.

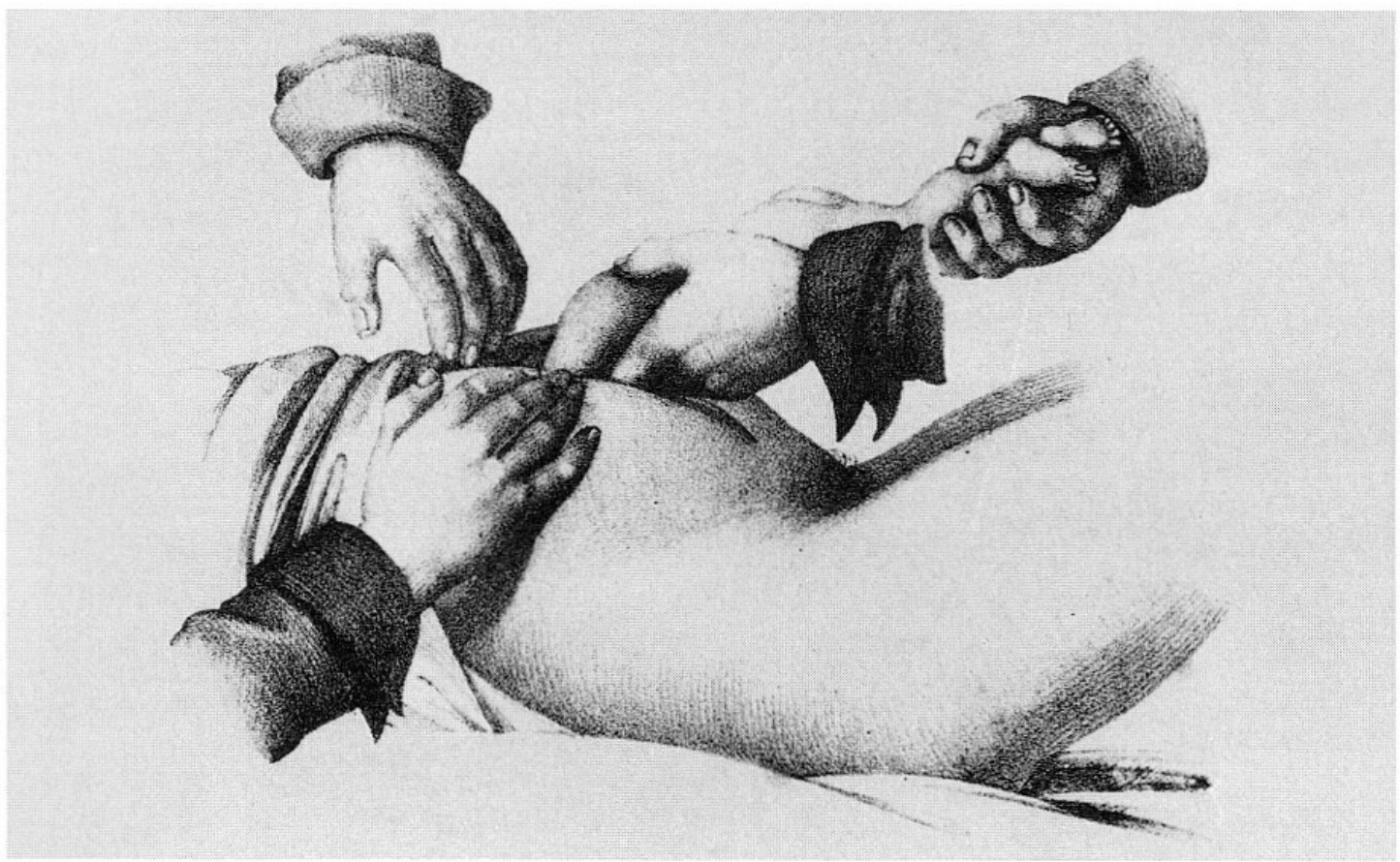

Abb. 5. Nach Eröffnung der Eihäute erfolgt das schnelle Hervorziehen des Kindes unter Vermeidung einer Einschnürung des Kopfes durch die Nabelschnur oder Einklemmen im Uterusmuskel. Siebolds Abbildungen, 1829 (wie Abb. 4), Tafel LIX. 
lenförmig vordrängende Blutung heraus; an Gefässunterbindung oder sonstige Blutstillung war nicht zu denken. Das Blut wurde so gut wie möglich mit Schwämmen abgetupft und der Uterus sich selbst zur Vereinigung überlassen; Locher hatte nämlich einige Jahre früher bei der Leichenöffnung einer acht Tage nach erfolgter Sectio caesarea verstorbenen Patientin die nur zusammengelegten Wundränder des Uterus fast ganz vereinigt gefunden. Den Schnitt durch Haut und Muskulatur verschloss er mit fünf blutigen Nähten, bedeckte die Wunde mit Charpie, etlichen Heftpflastern, einigen Kompressen und schliesslich mit einer breiten Leibbinde. Über das Befinden der Kreissenden während des Eingriffs gibt das Manuskript keinen Aufschluß; Locher betonte aber, wie gut seine Patientin den Eingriff überstanden hatte. Ein schmerzstillendes Mittel, dessen Hauptbestandteil «Laudanum liquidum Sydenhami» war, verabreichte Locher erst postoperativ ${ }^{26}$.

Bis zum Mittag des vierten auf die Operation folgenden Tages kam es unter Anwendung einiger innerlicher und äusserlicher Medikamente, Senfpflastern und Klysmata zu keinen nennenswerten Komplikationen. Die Wöchnerin schlief ziemlich ruhig und ass mit Appetit. Der Blutfluss aus dem Uterus ging durch die Genitalia externa ab, vom Verband wurden vorerst nur die oberen Teile erneuert. Am vierten Tag indessen erreichte Locher die Nachricht, die Frau liege im Sterben. Tatsächlich fand er sie in einem «misslichen Zustand» mit starrem Blick, kaltem Schweiss und bei getrübtem Bewusstsein. Nach Verabreichung von Riechmitteln und dem Einreiben krampfstillender Mittel zeigte sich gegen Abend eine Besserung. Locher führte die dramatische Veränderung auf die der glücklichen Geburt folgenden Emotionen zurück, entfernte den Säugling aus dem Krankenzimmer und verbot jeden Besuch. Von nun an ging es der Patientin täglich besser; einen Monat nach der Operation betreute sie das Kind selbst, in der siebten Woche stellte sich die Menstruation wieder ein, in der achten vermochte sie ihre Hausarbeit wieder zu besorgen. In der zwölften Woche erfreute den Arzt ein überraschender Besuch seiner Patientin mit ihrem Kind, ein «Beispiel von Gesundheit, Stärke und Schönheit». Über ein halbes Jahr lang widersetzte sich eine 5 bis $6 \mathrm{~mm}$ lange Wundöffnung jedem Heilungsprozess, um sich aber zuletzt doch zu verschliessen. Gegen Ende November 1817 riss die Wunde durch einen Brechanfall erneut $5 \mathrm{~mm}$ lang auf, und es traten Teile des Omentums in der Grösse einer mittleren Faust hervor. Locher reponierte mit Hilfe einer Löffelsonde mit grosser Geduld ein Stück nach dem andern und legte

26 Zur Zusammensetzung dieser offizinellen, opiumhaltigen Zubereitung vgl.z. B. das Dispensatorium Regium et Electorale Borusso-Brandenburgicum..., Berlin 1781, (Praeparata et composita), S. 50; Hagers Handbuch der pharmaceutischen Praxis..., hrsg. v. B. Fischer u. C. Hartwich, Bd. II, Berlin 1902, S. 522. 
eine blutige Naht an, die er mit Charpie und Heftpflaster bedeckte. Zwar vereinigten sich in der Folge die Wundränder wieder, doch blieb ein oberflächliches Geschwür zurück ${ }^{27}$.

\section{Nach allen Regeln der Kunst?}

Kurz nach 1800 standen den Geburtshelfern für die äussersten, verzweifelten Fälle eine ganze Reihe von speziellen Interventionen zur Verfügung: Neben dem Kaiserschnitt und den verkleinernden Operationen kannte man u. a. den Schambeinknorpelschnitt, die sogenannte Symphyseotomie, sowie die «künstliche Frühgeburt»; in weniger extremen Fällen kamen Wendeoperationen oder Zangen in Frage. Die einzelnen Indikationen für die speziellen Mittel und Methoden wurden sehr kontrovers diskutiert. Im Unterschied zu früheren Zeiten waren sich die Ärzte zwar weitgehend einig, dass der Arzt nicht die Pflicht habe, bei schwerer Geburt den Tod von Mutter oder Kind abzuwarten, um dann alles für den Überlebenden zu tun ${ }^{28}$. Ob es allerdings angebracht sei, der Mutter oder dem Kind die grösseren Risiken bzw. den Tod zugunsten des anderen zuzumuten, war umstritten. In Anbetracht der komplizierten Verhältnisse, die sowohl moralische Konflikte verursachten wie auch technische Unwägbarkeiten mit sich brachten, verwundert dies nicht. Hier können wir nur einige wenige Aspekte des gesamten Problemkreises andeuten, so dass zumindest deutlich wird, warum im vorliegenden Fall weder grössere Tatsachendivergenzen noch gewichtige moralische Konflikte auftraten, so dass der von Locher eingeschlagene Weg im grossen und ganzen von seinen Kollegen akzeptiert wurde.

Zunächst stand unbestreitbar fest, dass die Geburt ohne operativen Eingriff unmöglich zu einem glücklichen Ende kommen konnte. Die für die Mutter relativ risikoarme Perforation des kindlichen Kopfes ohne Embryotomie war bei einer «Conjugata» von «2, höchstens $2^{1} \frac{1}{2}$ Zoll» nicht mehr möglich. Nach damaliger Lehrmeinung war sicher, dass das Kind in einem solchen Fall nur durch eine (zusätzliche) Embryotomie durch die normalen Geburtswege

27 Locher (1818, wie Fussnote 25), S. 15, Ende November 1817. Das durch Kaiserschnitt entbundene Mädchen erreichte ein Alter von etwa sieben Jahren, wurde jedenfalls 1825 im Verzeichnis der Bürger und Ansässen der Stadt Zürich noch genannt, ist 1827 aber nicht mehr aufgeführt, also mit aller Wahrscheinlichkeit zwischenzeitlich verstorben.

$28 \mathrm{Zu}$ den ethischen Problemen der schweren Geburt vgl. z. B. Schulz, S.: Zur Ethik des geburtshilflichen Handelns bei Wilhelm Fabry von Hilden, in: Vorträge '93, hrsg. von der Stadt Hilden. Hilden (1993), S. 13-71 (= Hildener Museumshefte 5); ders. (1993, wie Fussnote 11) und ders.: Wenn Tod und Leben beieinanderstehen. Ethische, operative und institutionelle Aspekte zur Geschichte der Geburtshilfe, in: Kein Kinderspiel - das erste Lebensjahr, hrsg. vom Landschaftsverband Westfalen-Lippe, Münster 1994, S. 16-31. 
entbunden werden konnte. Das mütterliche Risiko bei diesem Eingriff hielten die meisten Ärzte (auf dem Kontinent) für ebenso hoch wie beim Kaiserschnitt ${ }^{29}$. Es bestand also nicht die konfliktträchtige Notwendigkeit, das geringere Risiko der Mutter (bei der Perforation) gegen das Leben des Kindes aufzurechnen. Die Diagnose des lebenden Kindes, die damals mit vielen Unsicherheiten belastet war $^{30}$, hatten die beiden Ärzte entsprechend dem damaligen Standard zweifelsfrei erhoben: sie konnten deutliche Bewegungungen des Kindes fühlen. Schliesslich hatten die Mutter und die Angehörigen in die von den Ärzten empfohlene Operation eingewilligt. Im Prinzip waren sich also alle an der Behandlung beteiligten Personen über das weitere Vorgehen einig. Locher und Spöndli gerieten damit nicht in die delikate Situation, die Frage nach der Berechtigung eines Eingriffes gegen den Willen der Mutter oder der Angehörigen beantworten zu müssen ${ }^{31}$. Die Schambeinfugendurchtrennung schliesslich, eine vom Mitte des 18. Jahrhunderts geborenen französischen Chirurgen Jean-René Sigault entwickelte Operation, schätzten viele deutschsprachige Geburtshelfer nicht als tatsächliche Alternative zum Kaiserschnitt ein. Insbesondere bezweifelten sie, ob man durch diesen Eingriff das knöcherne Becken wirklich entscheidend erweitern könne ${ }^{32}$. Die Frage, warum Locher trotz der diagnostizierten geringen Beckenmasse überhaupt versucht hat, eine Zangengeburt durchzuführen, lässt sich nicht eindeutig beantworten. Vielleicht liegt ein Grund darin, dass die Beckenmasse durch die Untersuchung mit der Hand nur unsicher bestimmt werden konnten.

In Grossbritannien wurde die Indikation für eine verkleinernde Operation allerdings etwas anders gestellt. Die englischen Chirurgen und Wundärzte bevorzugten oft auch bei sehr engen Becken (bis unter 13/4 Zoll) die Embryotomie und rieten vom Kaiserschnitt ab - auch wenn das Kind noch lebte. Diese Handlungsanzeige basierte auf einer anderen Beurteilung der zugrundeliegenden «Tatsachen», wie der Prognose der alternativen Eingriffe, und wurde charakteristischer Weise von dem moralischen Urteil begleitet, dass das Leben des Kindes dem Leben der Mutter unterzuordnen

29 Vgl. z. B. Osiander (1825, wie Fussnote 5), S. 197; Naegele (1845, wie Fussnote 5), S. 110-111.

30 Vgl. z. B. Porst, S.: Zur Geschichte der fetalen Herztonaufzeichnung in Deutschland, in: Zur Geschichte der Gynäkologie und Geburtshilfe, hrsg.v. L. Beck, Berlin, Heidelberg, New-York 1986, S. 119-132.

31 Viele Ärzte waren dagegen, einen Eingriff gegen den Willen der Mutter durchzuführen (vgl. Hufeland, C. W.: Vom Recht des Arztes über Leben und Tod, in: Journal der practischen Heilkunde 39 [1823], S. 3-28, Michaelis, 1833, wie Fussnote 5, S. 145; Naegele, 1845, wie Fussnote 5, S. 112). An Gegenstimmen fehlte es aber nicht (vgl. Osiander, 1821, wie Fussnote 17, S.325).

32 Vgl. Osiander (1821, wie Fussnote 17), S. 427 und S. 454-473; Naegele (1845, wie Fussnote 5), S. 171-181; vgl. auch Fasbender (1906, wie Fussnote 11), S. 864-873; Kloeppel (1992, wie Fussnote 11), S. 46-48. 
sei $^{33}$. Die Auszeichnungen, welche die ehrenwerte «Medical and Chirurgical Society of London» Locher angedeihen liess, belegen aber das Interesse an der kontinentalen Indikationsstellung. Inwieweit dafür moralische Motivationen oder nur eine pragmatische Aufgeschlossenheit ausschlaggebend waren, kann hier nicht weiter untersucht werden.

Während die Anzeige zum Kaiserschnitt in weitem Umfang moralische Entscheidungen verlangte, war das praktische Operieren ein pragmatisches Problem. Locher operierte in völliger Übereinstimmung mit den Prinzipien seiner Zeit. Der Schnitt durch die Linea alba, den Locher auswählte, wurde eindeutig als die beste Methode unter den bekannten Schnittrichtungen angesehen $^{34}$. Die Bauchdecken (mit verschiedener Technik) zu vernähen oder durch «Heft-Pflaster» zu verschliessen, war ebenso üblich wie die Uteruswunde offenzulassen. Zu Lochers Zeit diskutierte man zwar schon über die Uterusnaht, die Technik setzte sich aber erst ab den 1880er Jahren $\operatorname{durch}^{35}$.

\section{Zweiter Kaiserschnitt: Totgeburt und Tod der Mutter nach sieben Wochen}

Mitte Januar 1818 führten das Ausbleiben der Menstruation und heftige Bewegungen im Mutterleib zur Gewissheit, dass Elisabeth Bröckli aufs neue guter Hoffnung war. «Wünschenswerth wäre es gewesen», so schrieb Locher, «wenn sie nicht wieder schwanger geworden wäre. Allein, ändern lässt sich

33 Vgl. zu der unterschiedlichen Tatsacheneinschätzung z. B. Osiander (1825, wie Fussnote 5), S. 197: «Bei einer Verengerung bis auf $1 \frac{1}{2}$ Zoll noch an die Perforation und Zerstückelung zu denken, wie Osborn, Burns, Gardien es lehren, scheint mir ganz ungereimt zu seyn.», vgl. auch seine Hinweise auf Denman und Boer auf der S. 202/3; vgl. auch Naegele (1845, wie Fussnote 5), S. 111 sowie Osborn (1794, wie Fussnote 5), S. 154ff. Zur Diskussion um den «Wert» des mütterlichen bzw. kindlichen Lebens vgl. z. B. die typischen Argumentationen bei Osborn (1794, wie Fussnote 5), S. 135ff. u. 312ff. sowie Hufeland (1823, wie Fussnote 31) und Naegele (1826, wie Fussnote 17).

34 Vgl. Ritgen, F. A.: Die Anzeigen der mechanischen Hilfen bei Entbindungen, Giessen 1820, S. 447; Osiander (1825, wie Fussnote 5), S. 198; Michaelis (1833, wie Fussnote 5), S. 166; Busch (1838, wie Fussnote 21), S. 424; Kayser (1841, wie Fussnote 9), S. 116; Naegele (1845, wie Fussnote 5), S. 120; vgl. auch Fasbender (1906, wie Fussnote 11), S. 987-989.

35 Vgl. Ritgen (1820, wie Fussnote 34), S. 448; Osiander (1821, wie Fussnote 17), S. 368-369 (in besonderen Fällen hält er eine Naht der Gebärmutter aber für sinnvoll, vgl. S. 368); Michaelis (1833, wie Fussnote 5), S. 176-179; Naegele (1845, wie Fussnote 5), S. 126-127; vgl. auch Kehrer, F. A.: Ueber ein modifiziertes Verfahren beim Kaiserschnitt, in: Archiv für Gynäkologie 19 (1882), S. 177-209; Sänger, M.: Zur Rehabilitierung des classischen Kaiserschnittes. Nebst einem Anhang: Nachträge zur Geschichte der Uterusnaht beim Kaiserschnitte, in: Archiv für Gynäkologie 19 (1882), S. 370-399; Fasbender (1906, wie Fussnote 11), S. 991-994; Lehmann, V.: Zur Geschichte der Uterusnaht beim Kaiserschnitt, in: Zur Geschichte der Gynäkologie und Geburtshilfe, hrsg. v. L. Beck. Berlin, Heidelberg, New York 1986, S. 95-102. 
nichts, und so muss man mit Geduld den Ausgang der Sache erwarten $»^{36}$. Der Arzt liess die Frau einen Schnürgurt tragen und unterstützte die Wundränder mit Heftpflaster, denn er hegte grosse Bedenken, dass die Naht beim Anschwellen des Bauches platzen und die Därme heraustreten könnten. Die Schwangere behielt fortwährend ihre Munterkeit und äusserte den dringenden Wunsch, es möchten bei der kommenden Entbindung keine Versuche mit der Zange mehr unternommen, sondern sofort der Kaiserschnitt durchgeführt werden. Wiederum hing der Bauch über das Os pubis, ohne dass sich das Kind ins Becken gesenkt hätte. Vorsorglich liess Locher die Hochschwangere diesmal ins Spital einweisen.

Den Spitalakten ist zu entnehmen, dass sich hier ein Ereignis abspielte, das charakteristisch ist für das grosse medizinische Interesse, das der Fall mittlerweile fand. Die Patientin klagte, dass ihr ein fremder Arzt nach der Frage, ob sie die durch Kaiserschnitt glücklich entbundene Person sei, den Verband gelöst und das Pflaster teilweise entfernt habe. Locher hatte hierzu keinerlei Erlaubnis gegeben, und die Wundgschau-Kommission fand rasch heraus, dass es sich bei der fremden Person um Spitalarzt Ferdinand Adolf Stäbli ${ }^{37}$ von Brugg gehandelt hatte. In einem geharnischten Schreiben drückte ihm die Kommission ihr «Befremden und Missfallen» aus; gerade ein Spitalarzt hätte wissen müssen, dass ein solches Betragen in keinem Spital geduldet werden könne ${ }^{38}$. Stäbli schrieb der Behörde zerknirscht und reumütig zurück, billigte deren Reaktion und konnte nach eigenen Worten zu seiner Entschuldigung nur die «Liebe zu unserer Wissenschaft und Kunst» anführen $^{39}$.

Am 23. Mai 1818 nach sieben Uhr abends ging bei der Schwangeren plötzlich reines Blut durch die Vagina ab. Da der Kopf des Kindes, sogar die Sutura coronalis von aussen deutlich zu fühlen war, musste Locher mit einer Ruptur des Uterus rechnen. Die um zehn Uhr abends versammelten Ärzte kamen überein, den Kaiserschnitt ein zweites Mal zu wagen, was die Patientin sehr billigte. Gegen Mitternacht öffnete Locher den Bauch an der Stelle der Wundnaht; die sogleich erscheinende Menge koagulierten Blutes bewies tatsächlich die vor Stunden erfolgte Verletzung des Uterus. Beim Eindringen in die Eihäute zeigte sich, dass das siebeneinhalb Pfund wiegende Kind, des-

36 Locher, J. J.: Geschichte eines zweymal an der nämlichen Person verrichteten Kaiserschnitts, Mskr., Staatsarchiv Zürich W 52.11.2, Mitte Januar 1818, o. J. [1818], S. 19.

37 Ferdinand Adolf Stäbli (1772-1835), praktischer Arzt in Brugg, 1810-1821 Spitalarzt in Königsfelden, operierte 1827 Heinrich Pestalozzi erfolglos, 1832-1835 eidgenössischer Oberfeldarzt.Vgl. Winzenried, M.: Das Militärsanitätswesen in der Schweiz von der Mediation bis zum Sonderbundskrieg, Basel 1954, S. 65-67.

38 Brief vom 12.5.1818, Acta der Wundgschau 1817-1832, Bd. 8, Staatsarchiv Zürich H I 334, S. 28.

39 Ibid., S. 29. 


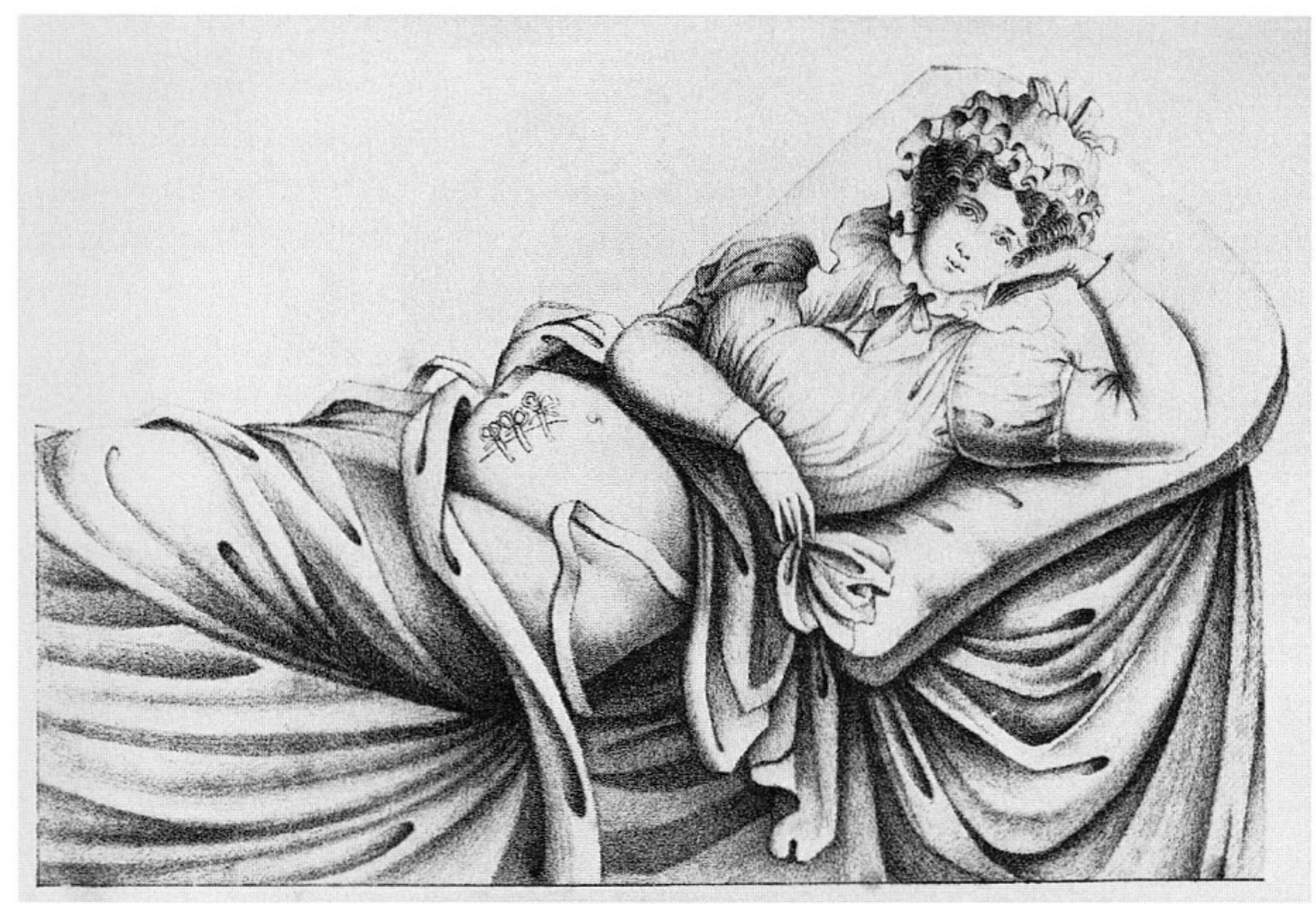

Abb. 6. Die Wundränder der Bauchdecke werden durch blutige Nähte mit breiten Bändchen zusammengehalten. Siebolds Abbildungen, 1829 (wie Abb. 4), Tafel LX.

Abb. 7. Rachitisch plattes Becken der 1817 und 1818 von Johann Jakob Locher zweimal durch Kaiserschnitt entbundenen Patientin. Medizinhistorisches Institut und Museum der Universität Zürich, Inv.-Nr. 6476.

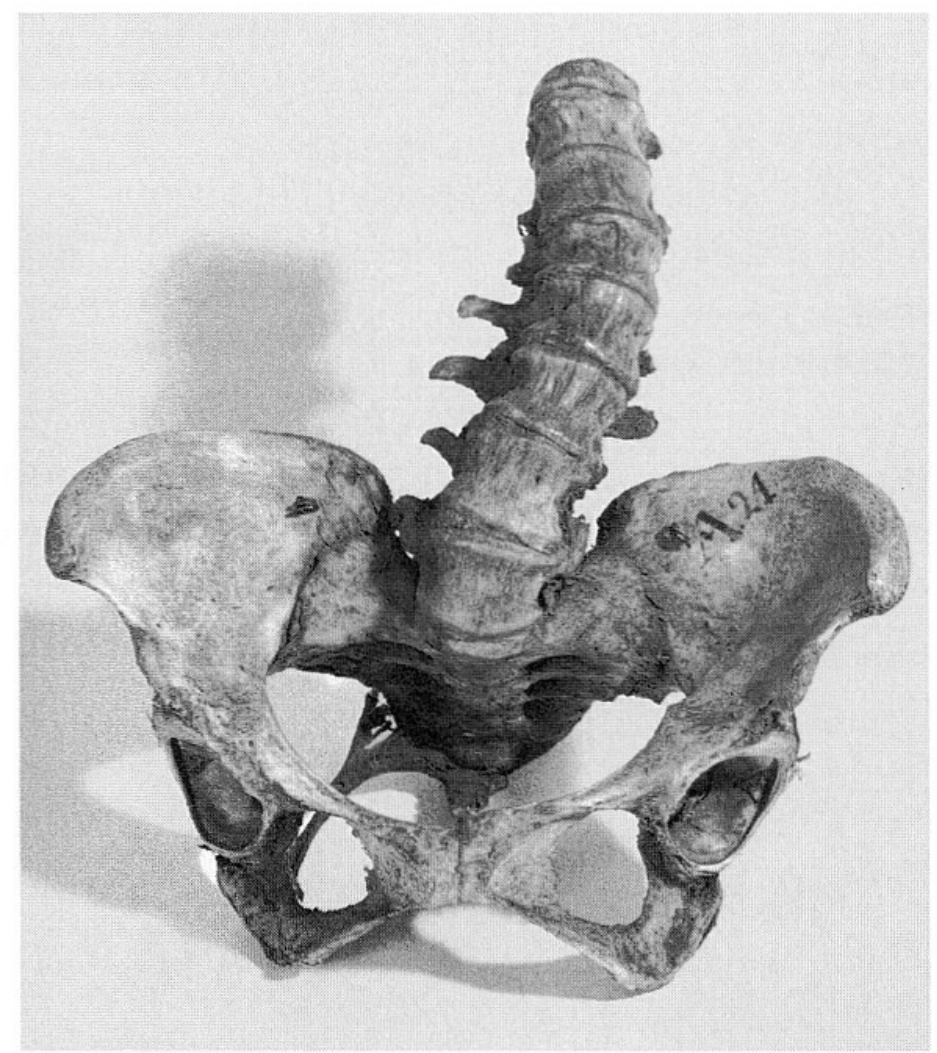


sen Hals von der Nabelschnur umwickelt war, kein Lebenszeichen mehr gab. Locher legte vier blutige Nähte an und unterstützte die Wunde wiederum mit Charpie und Heftpflaster. Trotz einigem Bedauern über die Totgeburt blieb die Patientin vorerst bei guten Kräften, froh, von schwerer Last befreit zu sein. Am folgenden Tag stellten sich Krämpfe im ganzen Unterleib ein, der Puls war unregelmässig und schwach, der Bauch schmerzhaft aufgetrieben. Locher missfiel besonders, dass die Patientin erstmals deprimiert wirkte und sich unzufrieden über ihr schweres Schicksal äusserte. Trotz wiederholt angewandter innerlicher Medikation und zahlreicher Klistiere blieb ihr Zustand die nächsten Tage fortwährend kritisch. Zwar beurteilte Locher gemäss den medizinischen Auffassungen der Zeit die Suturen als «sehr schön, rein und mit gutem Eiter bedeckt $»^{40}$. Die Zeichen einer fortschreitenden Sepsis waren aber nicht zu übersehen. Eine Wundrose breitete sich anfangs Juni, von der Operationswunde ausgehend, nach und nach über den ganzen Körper aus und verursachte - begleitet von ständigem Fieber - der Frau ein fast unerträgliches Hitzegefühl. «Kühlende» Arzneimittel und Diät wurden fortgesetzt, ohne das Leiden der Wöchnerin nachhaltig zu mildern: «Man bedenke nur! Über den ganzen Rüken ein Erysipelas, und auf dem Bauch eine solche Wunde! ${ }^{41}$ Bis zum 24 . Juni blieb der Zustand unverändert ernst; jetzt schien sich vorübergehend eine leichte Besserung einzustellen. Die Frau ass wieder mit einigem Appetit, eine gewisse Munterkeit des Geistes kehrte zurück. Die nur teilweise zugeheilten Wundränder bildeten ein ununterbrochen nässendes fistulöses Geschwür von der Grösse eines Groschens und liessen wiederum eine Ausstülpung des Omentum oder der Gedärme befürchten. Wirklich zeigte sich am 29. Juni ein kleines Stück Darm, das aber den sonst recht guten Heilungsprozess der Wundränder nicht gross zu beeinflussen schien. Die Kranke blieb dank kleinen Gaben von Opium weiterhin recht munter. Am 7. Juli klagte sie aber unverhofft über einen schrecklichen Schmerz im Unterleib und erbrach sich bald darauf heftig. Nach zwei verhältnismässig ruhig verbrachten Tagen verstarb die Patientin am Morgen des 9. Juli 1818 um viertel nach sieben trotz Analeptika, Riechmitteln und weiteren Belebungsversuchen, möglicherweise an einem Darmverschluss.

Die am Nachmittag des folgenden Tages vorgenommene Sektion zeigte die Bauchwunden bis auf die noch immer offene Stelle vernarbt. An den inneren Organen beobachtete Locher heftige Entzündungen und sogar einzelne brandige Stellen, die für ihn die vorherigen Symptome der Wundinfektion und den doch eher überraschend schnellen Tod erklärlich machten.

40 Locher, J. J.: Geschichte eines zweymal an der nämlichen Person verrichteten Kaiserschnitts, Mskr., Staatsarchiv Zürich W 52.11.2, 2. Juni 1818, o. J. [1818], S. 31.

41 Ibid., S. 32, 4. Juni 1818. 
Der Uterus erwies sich als «beynahe auf dem natürlichen Zustand zusammengezogen $»^{42}$ und vollkommen geheilt bis auf eine kleine, rundliche Öffnung von der Grösse eines Mandelkerns; diese schien eine Folge der ersten Kaiserschnittoperation gewesen zu sein und trug die Schuld an der plötzlichen Verletzung des Uterus kurz vor dem zweiten Eingriff. Die Gestalt des freipräparierten Beckens gab Locher vollends Aufschluss über die zweimalige Unmöglichkeit, die Schwangere komplikationslos zu entbinden.

\section{Künstliche Frühgeburt?}

Die erneute Schwangerschaft von Elisabeth Bröckli stand im Januar 1818 fest. Die Menstruation war bereits einige Monate ausgeblieben, der frühere Schwangerschaftsverlauf liess Böses ahnen. Musste in dieser Situation wirklich die Geburt abgewartet werden? Gab es keine Möglichkeit, die drohenden Komplikationen durch einen rechtzeitigen Eingriff abzuwenden? Warum hatte Locher nicht darauf gedrängt, eine künstliche Frühgeburt einzuleiten? Dieser Eingriff war bereits im 18. Jahrhundert in Grossbritannien entwickelt und schon 1799 vom Heidelberger Geburtshelfer Anton Mai (1742-1814) propagiert worden. Die künstliche Frühgeburt wurde meist im siebten Schwangerschaftsmonat durch einen Eihautstich oder eine Dehnung des Gebärmuttermundes ausgelöst ${ }^{43}$. Hätte dieser Eingriff nicht die Chance geboten, Mutter und Kind zu retten? Locher erwähnt diese Operation mit keinem Wort. Doch dies muss nicht bedeuten, dass er nicht lege artis gehandelt hat - möglicherweise kannte er diese Technik noch nicht. Bekannt ist jedenfalls, dass sie erst durch die im Jahre 1818 veröffentlichte Monographie über die «Künstliche Frühgeburt» des in Frankfurt am Main tätigen Geburtshelfers Carl Wenzel (1769-1827) (4 $^{4}$ und - in besonderem Masse - durch die 1820 erschienenen «Anzeigen der mechanischen Hilfen bei Entbindungen» des Giessener Geburtshelfers Ferdinand A. Ritgen (1787-1867) ${ }^{45}$ in den Blick der breiten Öffentlichkeit gelangte.

42 Ibid., S. 44, 9. Juli 1818.

43 Zur Durchführung der künstl. Frühgeburt vgl. Wenzel, C.: Allgemeine geburtshilfliche Betrachtungen und über die künstliche Frühgeburt, Mainz 1818, S. 127-213; Ritgen (1820, wie Fussnote 34), S. 435-440; Osiander (1821, wie Fussnote 17), S. 473-495; Naegele (1845, wie Fussnote 5), S. 182-207. Kritische Stimmen meldeten sich allerdings von Anfang an zu Wort (vgl. z. B. Osiander 1821, wie Fussnote 17, S. 473-495).

44 Wenzel (1818, wie Fussnote 43).

45 Ritgen (1820, wie Fussnote 34); zur Bewertung des Bekanntheitsgrades der künstlichen Frühgeburt vgl. Naegele (1845, wie Fussnote 5), S. 181-207, bes. S. 182/3; vgl. auch Fasbender (1906, wie Fussnote 11), S. 849-864; Kutzer, M.: Über Natur und Kunst in der Arzneiwissenschaft. Carl Wenzel (1769-1827) als Lehrer und Kritiker der Geburtshilfe seiner Zeit, in: Medizinhistorisches Journal 20 (1985), S. 391-416; Kloeppel (1992, wie Fussnote 11), S. 54-56. 


\section{Beckenbeschreibung}

Schon anlässlich seines Referats vor der Medizinisch-chirurgischen Kantonalgesellschaft in Zürich hatte Stadtarzt Locher seinen Kollegen eine Skizze des von ihm präparierten Beckens vorgelegt. Das Becken selbst nutzte er später wohl zu Lehrzwecken am Medizinisch-chirurgischen Institut; es vererbte sich nach seinem Tod von 1832 an seinen Neffen Heinrich LocherZwingli, dem ersten Professor für Chirurgie der 1833 gegründeten Zürcher Hochschule. Von diesem gelangte das Objekt in den Besitz der Zürcher Frauenklinik, von wo es 1983 mit 29 weiteren Beckenpräparaten ans Medizinhistorische Museum der Universität Zürich überging ${ }^{46}$.

Das Präparat besteht aus einem hochgradig rachitisch platten Becken und einem stark verkrümmten Wirbelsäulenabschnitt, der beim angesägten neunten Brustwirbel beginnt. Bindegewebe und Knorpelsubstanz sind teilweise erhalten. Leider sind die beiden Oberschenkelknochen, die ein früheres Protokoll erwähnt, nicht mehr vorhanden ${ }^{47}$. Die ganze Wirbelsäulenpartie beschreibt oberhalb des Sacrums in einem Winkel von $30 \mathrm{Grad}$ eine linkskonvexe Lumbalskoliose mit dem Scheitelpunkt auf Höhe des zweiten Lendenwirbels. Ausserdem vollführt sie eine Achsendrehung, im unteren Verlauf nach links, im oberen kompensatorisch wieder nach rechts. Hinzu kommt eine Hyperlordose mit einem Kreuzbeinbasiswinkel von etwa $80 \mathrm{Grad}$; das Kreuzbein stellt sich als sogenanntes Sacrum acutum beinahe horizontal. Alle diese Veränderungen zeugen deutlich von einer massiven rachitischen Knochenerweichung in frühester Kindheit. Unter der Last des Körpergewichts sank das Promontorium tief in das Becken hinein, worauf die zunehmende Lordose eine einigermassen aufrechte Körperhaltung zu erhalten versuchte. Der erste Sakralwirbel ist ganze 11,5 cm breit, doch fehlt die sonst bei Rachitis übliche Krümmung des Kreuzbeins. Dafür knickt das Steissbein im spitzen Winkel in das kleine Becken ab, möglicherweise infolge einer intravitalen Verletzung.

Das rechte Hüftbein steht etwas tiefer als das linke, Hinweise auf eine Beckenneigung fehlen. Beide Darmbeinschaufeln blieben im Wachstum zurück; sie sind flach und klaffen in der Distantia cristarum $27 \mathrm{~cm}$, in der Distantia spinarum $21 \mathrm{~cm}$; die Gelenkpfannen richten sich nach vorn. Die Sitzbeinknorren rückten so weit auseinander, dass sich der Schambogen nun

46 Damals war Urs Boschung Konservator des Medizinhistorischen Museums der Universität Zürich.

47 Altes Inventar der Beckensammlung der Universitäts-Frauenklinik Zürich, Typoskript, Zürich o. J., Medizinhistorisches Institut und Museum der Universität Zürich. Vgl. auch Inventarkartei Nr. 6476 des Medizinhistorischen Museums der Universität Zürich. 
über $100 \mathrm{Grad}$ spreizt. Statt eines Ovals bildet der Eingang des kleinen Beckens eine Herzform: Der Querdurchmesser beträgt zwar $13 \mathrm{~cm}$, dagegen verkürzt sich die Conjugata vera durch das stark vorspringende Promontorium auf $7 \mathrm{~cm}$. Dieser enge Geburtskanal verunmöglichte den Durchtritt eines Kindes. Spätestens die nur $16 \mathrm{~cm}$ lange Conjugata externa muss die geburtsverhindernde Beckenanatomie aufgedeckt haben.

Die Körperhaltung der Patientin war aufgrund der starken Lordose von einem Hängebauch geprägt, während das Gesäss beinahe horizontal nach hinten auslud. Nebst allen andern Deformitäten, die eine derart gravierende Rachitis charakterisieren, zeigten die Beine und Füsse sicherlich typische korkzieherartige Verdrehungen und massive Verkürzungen, was die Gehfähigkeit drastisch eingeschränkt haben muss ${ }^{48}$.

\section{Erster akademisch gebildeter Stadtschnittarzt von Zürich}

Als Johann Jakob Locher 1813 zum Amt des Stadtschnittarztes und Kantonswundarztes gelangte, war er der erste akademisch gebildete Mediziner an dieser bisher den Wundärzten vorbehaltenen obersten Chirurgenstelle des Zürcher Stadtstaates. Dem Stadtschnittarzt war die chirurgische Betreuung der Spitalpatienten ebenso übergeben wie eine Lehrstelle am Medizinisch-chirurgischen Institut mit zwei klinischen Unterrichtsstunden. Er wurde zur Prüfung der angehenden Chirurgen und Hebammen beigezogen und hatte ausserdem Sitz und Stimme im kantonalen Sanitätsrat ${ }^{49}$.

Johann Jakob Locher wurde am 27. Dezember 1771 in Zürich als Spross einer seit 1551 in Zürich eingebürgerten, angesehenen Ratsfamilie geboren. Sein Grossvater und ebenso sein Vater ${ }^{50}$ hatten sich neben ihrer chirurgischen Tätigkeit noch dem Barbierhandwerk gewidmet und gehörten der Gesellschaft der Ärzte und Wundärzte innerhalb der Zunft zur Schmiden an. Noch während des Besuchs des höheren Gymnasiums erhielt er den chirurgischen Lehrbrief und vom Vater die erste Einführung in dessen Spital- und Privatpraxis. Dem Besuch des Medizinisch-chirurgischen Instituts schloss sich ein Studium an der Universität Jena an, wo Justus Loder und Christoph Wilhelm Hufeland als seine meistgeschätzten Lehrer wirkten. 1792 verfasste

48 Die Autoren danken Dr. med. Iris Ritzmann vom Medizinhistorischen Institut und Museum der Universität Zürich für ihr Gutachten.

49 Wehrli, G. A.: Die Krankenanstalten und die öffentlich angestellten Aerzte und Wundärzte im alten Zürich, in: Mittlg. Antiquarische Ges. Zürich Bd. 31.3, Zürich 1934, S. 50-56.

50 Neujahrsblatt von der Gesellschaft auf der Chorherrenstube an die lernbegierige Zürcherische Jugend auf das Neujahr 1808, o. O. [Zürich] 1808. 
Locher eine Dissertation über die Operation der Hasenscharte ${ }^{51}$ und besuchte zur weiteren Ausbildung Berlin und Würzburg. 1793 in seine Heimatstadt zurückgekehrt, wirkte er als Gehilfe seines Vaters und erhielt bei dessen Tod 1807 das Amt eines Spitalarztes, bevor er ab 1813 als Stadtschnittarzt die höchste chirurgische Stellung im Kanton bekleidete. 1820 bis 1830 gehörte er als Abgeordneter der Zunft zur Schmiden auch dem Grossen Rat an, ohne sich nachhaltig um sein Parlamentsmandat zu kümmern. Rasch erfreute sich Locher eines guten Rufes als geschickter Operateur und Geburtshelfer, der grossen Wert auf therapeutische Einfachheit und Sorgfalt legte. Mit besonderer Genauigkeit besorgte er die Verbände und kümmerte sich auch intensiv um die Qualität seiner Instrumente, um dadurch die Dauer der Eingriffe zu verkürzen und die Operationsschmerzen zu vermindern. Schwierigen Eingriffen stand Locher mit Skepsis gegenüber und galt als erklärter Feind der Trepanation. Mit dem Lesen medizinischer Schriften, überhaupt mit dem Studium neuerer Erkenntnisse seiner Wissenschaft gab er sich kaum ab, besass aber einiges Interesse an der Naturgeschichte und eine entsprechende Sammlung ${ }^{52}$. Als Lehrer am Medizinisch-chirurgischen Institut vermochte Locher nach dem Zeugnis eines seiner Studenten wenig zu überzeugen: «Accouchement liest Herr Stadtarzt Locher so unfleissig, dass es besser wäre, er würde gar nichts lesen; zudem ist er gar nicht wohlbestellt, sobald er in das Feld der theoretischen Geburtshülfe hinein kommt.» ${ }^{53}$

Gemäss den Schilderungen eines zeitgenössischen Biographen und Kollegen galt Locher als Persönlichkeit von «geselligem, offenen, aber nothfestem Charcter» und besass eine ungemeine Lebhaftigkeit von Körper und Geist. «Aus Kleinigkeiten konnte er in gesellschaftlichem Leben ein grosses machen und denselben einen Anstrich geben, dass man beynahe zu glauben berechtigt wurde, noch nie etwas so gehört zu haben, welchem dann seine Bassstimme und seine flammenden, alles ausspähenden Augen noch mehr Gewicht gaben. Daneben war er dann gefühlvoll, welche Eigenschaft auch durch den vieljährigen gewohnten Anblick des Leidens in allen Gestalten niemals so weit abgestumpft werden mochte, dem Unglücklichen ein tiefes Mitgefühl, seine wohlthätigste Theilnahme, ja selbst Thränen des Mitleids

51 Locher, J. J.: Dissertatio inauguralis sistens cogitatio quaedam de labii leporini operatione, Jena 1792.

52 Anonymus [Locher-Balber, H.]: Nachruf auf J. J. Locher, in: Verh. Schweiz. Naturf. Ges. in Genf, Nr. 17, Genf 1832, S. 137-139.

53 Bezel, E.: Johann Jakob Steger (1798-1857), Beispiel eines Medizinstudiums im frühen 19. Jahrhundert nach den Briefen an seine Eltern, Med. Diss., Zürcher med. gesch. Abh. 147, Zürich 1981, S. 77. 
zu versagen. ${ }^{54}$ Johann Jakob Locher war verheiratet mit Anna Regula Hofmeister (1773-1828), die die Apotheke zur Wassermühle und die von einigen Gesellen betriebene Barbierstube beaufsichtigte. Da das Ehepaar kinderlos blieb, nahm es einen Knaben von Lochers Bruder auf, den es seit dem zehnten Lebensjahr an Kindes Statt erzog und zum Arzt ausbilden liess. Nach dreijährigem Krankenlager verstarb Locher am 15. Januar 1832 an einem Lungentumor, nur ein gutes Jahr bevor durch die Gründung der Zürcher Hochschule und damit einer Medizinischen Fakultät das Amt des Stadtschnittarztes aufgehoben wurde ${ }^{55}$. Er hatte nicht mehr die Genugtuung, die Wahl seines Neffen Heinrich Locher-Zwingli zum ersten Professor und Direktor der chirurgischen Klinik mitzuerleben ${ }^{56}$.

54 Meyer-Pestalozzi, J. L.: Biographie auf J. J. Locher, Mskr., o. J. [1832], Porträt- und Biographiensammlung «Meyer zum Felsenegg», Medizinhistorisches Institut und Museum der Universität Zürich.

55 Callisen, A. C. P.: Medicinisches Schriftsteller-Lexicon, Bd. 11, Kopenhagen 1832, S. 423f., Bd. 30, Kopenhagen 1842, S. 95. Garnaus, A.: Die Familie Locher von Zürich, Zürich 1924, S. 89f. Historisch-Biographisches Lexikon der Schweiz, Bd. 4, Neuenburg 1927, S. 698. Milt, B.: Geschichte des Zürcher Spitals, in: Zürcher Spitalgeschichte, Bd. 1, Zürich 1951, S. 56. Leisibach, M.: Das Medizinisch-chirurgische Institut in Zürich 1782-1833, Zürich 1982, S. 88.

56 Rahn-Escher, C.: Prof. Dr. Heinrich Locher, eine biographische Skizze, Zürich 1865. Muralt, R. H. von: Heinrich Locher, eine lokalhistorische Betrachtung der Entwicklung der Chirurgie in Zürich, NZZ, 21.8.1945. Steiner-Metzger, U.: Der Chirurg Heinrich Locher-Zwingli (1800-1865), Med. Diss., Zürcher med. gesch. Abh. 244, Zürich 1992. 